\title{
Identification of the anteroposterior and mediolateral position of lion paws and tracks using 3D geometric morphometrics
}

\author{
Antoine F.J. Marchal ${ }^{1,2 *}(\mathbb{D})^{\S}$, Philippe Lejeune ${ }^{2}$ \& P.J. Nico de Bruyn ${ }^{1}$ \\ ${ }^{1}$ Mammal Research Institute, Department of Zoology and Entomology, Faculty of Natural and Agricultural Sciences, \\ University of Pretoria, Private Bag X20, Hatfield, Pretoria, 0028 South Africa \\ ${ }^{2}$ Forest Resources Management, Department of Biosystems Engineering (BIOSE), Gembloux Agro-Bio Tech (GxABT), \\ 2. Passage des Déportés, B-5030, Gembloux, Belgium \\ Received 26 September 2016. To authors for revision 22 November 2016. Accepted 22 March 2017
}

\begin{abstract}
Estimating the distribution and status of animal populations is crucial in various fields of biology. Monitoring species via their tracks is controversial due to unreliable recording techniques, manipulator bias and substrate variation. Furthermore, subjective identification of the foot that produces each track can lead to significant errors, for example, when assigning tracks made by different feet from the same individual to different individuals. The aim of this research was to develop an accurate, consistent and objective algorithm to identify the anteroposterior (hind/front) and mediolateral (right/left) position from digital threedimensional (3D) models of African lion (Panthera leo) paws and tracks using geometric morphometrics. We manually positioned 12 fixed landmarks on 132 paws and 182 tracks recorded in 3D using digital close-range photogrammetry. We used geometric morphometrics to evaluate and visualize the shape variation between paws and between tracks along the anteroposterior and mediolateral axes, and between paws and tracks. The identification algorithm using linear discriminant analysis with jack-knifed predictions reached a maximum accuracy of $95.45 \%$ and $91.21 \%$ for paws and tracks, respectively. We recommend the use of this objective position identification algorithm in future studies where tracks are compared between individual African lions.
\end{abstract}

Key words: digital 3D model, foot, footprint, anteroposterior, mediolateral, geometric morphometrics, Panthera leo, photogrammetry, tracking.

\section{INTRODUCTION}

Human activities may well be the cause of a sixth biodiversity mass extinction (Ceballos et al., 2015), which particularly affects carnivores (Cardillo et al., 2004). Assessing spatial and temporal trends of animal populations is the keystone of any conservation efforts (Yoccoz, Nichols \& Boulinier, 2001). In the absence of direct observations, tracks offer a non-invasive, low-cost approach to gain information on elusive species such as carnivores (Heinemeyer, Ulizio \& Harrison, 2008). For more than three decades, the monitoring of tigers (Panthera tigris) in India was based on the 'pugmark census method' (Karanth et al., 2003; Sharma, Jhala \& Sawarkar, 2005). Tracings or plaster casts of tracks made by the

*To whom correspondence should be addressed. E-mail: marchal.ant@gmail.com

${ }^{8}$ ORCID: 0000-0002-9486-2183 hind left paws were sampled countrywide for individual tiger identification (Karanth et al., 2003; Sharma et al., 2005). Besides a poor analytical framework and subjective identification, this method received criticism for ignoring the variation related to different manipulators and substrates (Karanth et al., 2003). Furthermore, the misidentification of the foot from which each track originates led to substantial errors (e.g. by assigning tracks made by different feet of the same individual to different individuals) (Karanth et al., 2003). Other studies aimed at objectively identifying individuals using measurements extracted from specific tracks (e.g. hind or hind left tracks) of felids such as cheetahs (Acinonyx jubatus; Jewell et al., 2016), mountain lions (Puma concolor; Smallwood \& Fitzhugh, 1993; Grigione, Burman, Bleich \& Pierce, 1999; Jewell, Alibhai \& Evans, 2014), snow leopards (Panthera uncia; Riordan, 1998) and tigers (Riordan, 1998; Sharma et al., 2005). In 
this article, we aim to illustrate an accurate and consistent approach to objectively identify the anteroposterior (front or hind, a.k.a. manus or pes) and mediolateral (right or left) position from digital three-dimensional (3D) models of African lion (Panthera leo) paws and tracks using landmarkbased geometric morphometrics.

\section{MATERIALS AND METHODS}

Paw and track sampling using photogrammetry

Lion paws and tracks were sampled in the Hluhluwe-iMfolozi ( 900 $\left.\mathrm{km}^{2}\right)$ and Tembe Elephant Parks $\left(\sim 300 \mathrm{~km}^{2}\right)$ both located in the KwaZuluNatal (KZN) Province, South Africa, and managed by Ezemvelo KZN Wildlife (EKZNW). We opportunistically sampled 132 paws (front right (FR), front left $(F L)$, hind right $(H R)$ and/or hind left $(H L)$ ) from eight adult females, four adult males, five subadult females, 14 subadult males, three juvenile females and five juvenile males during nocturnal captures (Table 1). All lions were captured as part of routine management operations that were unrelated to this project and conducted by EKZNW's staff, which included a qualified wildlife veterinarian.

We recorded a total of 182 tracks from 12 different trails (Table 2). A trail was defined as a continuous sequence of tracks belonging to one individual (Liebenberg, Louw \& Elbroch, 2010). Our shortest trail contained seven tracks while the longest contained 31 tracks. Tracks that belonged to both male/female and adult/juvenile individuals were sampled in riverbeds and on dirt roads after direct observations or in front of camera traps (Cuddeback Attack, Green Bay, Wisconsin, U.S.A.) (Table 2). The camera traps $(n=6)$, which were checked daily, were strategically located along game paths and near waterholes. The foot from which each track originates (i.e. FR, FL, HR or $\mathrm{HL}$ ) was identified by the track's relative position within the sequence (Liebenberg, 1990; Liebenberg et al., 2010). All trails were less than 24 hours old and were characteristic of an overstep walk where the hind foot registered beyond the front track (Liebenberg, 1990; Liebenberg et al., 2010). Thirty tracks (11 FR, 13 FL, $4 \mathrm{HR}$ and $2 \mathrm{HL}$ ) from the trails were missing (i.e. destroyed by other tracks) or discarded due to poor quality.

As described in Marchal, Lejeune \& De Bruyn (2016), we took between 9 and 15 overlapping photographs at different angles and distances from the object of interest (i.e. paw or track) with a scale bar. Two digital single-lens reflex cameras were used for the sampling: Nikon D7100 (24.1 megapixels) with Nikkor $18-70 \mathrm{~mm}$ f/3.5-4.5 and Nikon D80 (10 megapixels) with Nikkor $50 \mathrm{~mm}$ f/1.8 (Nikon Corporation, Tokyo, Japan). We used the photogrammetric package Agisoft PhotoScan Professional Edition version 1.1 .4 build 2021 (Agisoft LLC, Saint Petersburg, Russia) to process digital 3D meshes. Masking the photographs was applied for the paws but not for the tracks, and we used both auto-calibration and optimization in the camera alignment step (Marchal et al., 2016). The scale bar enabled scaling of the 3D models. Close-range digital photogrammetry applied to hominid and human tracks showed comparable results to optical laser scanning (Vi900 KonicaMinolta) (Bennett, Falkingham, Morse, Bates \& Crompton, 2013). Accuracy tests on the Vi900 Konica-Minolta yielded an accuracy of $0.19 \mathrm{~mm}$ on $x$-plane, $0.14 \mathrm{~mm}$ on $y$-plane and $0.10 \mathrm{~mm}$ on $z$-plane (Keating, Knox, Bibb \& Zhurov, 2008).

\section{Landmark-based geometric morphometrics}

Twelve fixed landmarks were digitized on the 3D meshes of paws (Fig. 1a) and tracks (Fig. 1b) using the Geomorph package (Adams \& OtárolaCastillo, 2013; Adams, Collyer \& Sherratt, 2015) in the R program (R Development Core Team, 2014). Landmarks are discrete endpoints that are biologically homologous anatomical loci (Zelditch, Swiderski \& Sheets, 2012). We only used landmarks type II (i.e. curvature extrema of a local structure) (Bookstein, 1991) that were manually positioned at the extremes of the two outer bottom lobes and the two top pseudo-lobes of the main pad, and the two extremes located on the longest axis of each toe (Fig. 1a,b). These 12 landmarks were selected amongst the 25 landmarks chosen by Jewell et al. (2014) and Jewell et al. (2016). They are easy to locate and we believe that they represent the minimum number of landmarks needed to fulfil the objective of this research. We used the same landmark digitization sequence for all paws and tracks by taking into consideration rotation symmetry between them (Fig. 1a,b). To assess repeatability of the manual landmark digitization, we selected 10 paws and 10 tracks on which the landmarks were positioned four times, on different days, by five different manipulators with no prior experience in digitization. This subsample was representative of our database and complete for testing the digitization repeatability.

We used geometric morphometrics to extract features from paws and tracks. Geometric morphometrics involved a generalized Procrustes analysis 
Table 1. Details of the sampled paws. Lions sampled in Hluhluwe-iMfolozi Park and Tembe Elephant Park have unique identifications (i.e. ID) that begin with 'HiP' and 'Tembe', respectively. 'FL' = front left, 'FR' = front right, 'HL' = hind left and 'HR' = hind right.

\begin{tabular}{|c|c|c|c|c|c|c|c|}
\hline Age & Sex & Individual ID & $\mathrm{FL}$ & FR & $H L$ & $\mathrm{HR}$ & Total \\
\hline Adult & Female & HiP 309 & 1 & & 1 & 1 & 3 \\
\hline Adult & Female & HiP 311 & & 1 & 1 & 1 & 3 \\
\hline Adult & Female & HiP 404 & 1 & & 1 & & 2 \\
\hline Adult & Female & HiP 406 & 1 & & 1 & 1 & 3 \\
\hline Adult & Female & HiP 618 & 1 & & 1 & 1 & 3 \\
\hline Adult & Female & HiP 618 & 1 & 1 & 1 & 1 & 4 \\
\hline Adult & Female & HiP 622 & & 1 & 1 & 1 & 3 \\
\hline Adult & Female & HiP 625 & & & 1 & 1 & 2 \\
\hline Adult & Female & HiP 636 & 1 & 1 & 1 & 1 & 4 \\
\hline Adult & Male & HiP 312 & 1 & & 1 & 1 & 3 \\
\hline Adult & Male & HiP 405 & & & 1 & 1 & 2 \\
\hline Adult & Male & HiP 626 & & 1 & 1 & 1 & 3 \\
\hline Adult & Male & Tembe 5 & 1 & 1 & 1 & 1 & 4 \\
\hline Subadult & Female & HiP 627 & & 1 & 1 & 1 & 3 \\
\hline Subadult & Female & HiP 631 & 1 & & 1 & & 2 \\
\hline Subadult & Female & HiP 633 & & & 1 & 1 & 2 \\
\hline Subadult & Female & HiP 634 & & 1 & 1 & 1 & 3 \\
\hline Subadult & Female & HiP 641 & 1 & 1 & 1 & 1 & 4 \\
\hline Subadult & Male & HiP 527 & 1 & 1 & 1 & 1 & 4 \\
\hline Subadult & Male & HiP 619 & & 1 & 1 & 1 & 3 \\
\hline Subadult & Male & HiP 628 & 1 & 1 & 1 & 1 & 4 \\
\hline Subadult & Male & HiP 629 & 1 & & 1 & 1 & 3 \\
\hline Subadult & Male & HiP 635 & 1 & & 1 & 1 & 3 \\
\hline Subadult & Male & HiP 640 & & 1 & 1 & 1 & 3 \\
\hline Subadult & Male & HiP 718 & 1 & 1 & 1 & 1 & 4 \\
\hline Subadult & Male & HiP 730 & 1 & 1 & 1 & 1 & 4 \\
\hline Subadult & Male & HiP 731 & 1 & 1 & 1 & 1 & 4 \\
\hline Subadult & Male & HiP 732 & 1 & & 1 & 1 & 3 \\
\hline Subadult & Male & HiP 733 & 1 & 1 & 1 & 1 & 4 \\
\hline Subadult & Male & HiP 734 & 1 & 1 & 1 & 1 & 4 \\
\hline Subadult & Male & HiP 735 & 1 & 1 & 1 & 1 & 4 \\
\hline Subadult & Male & Tembe 4 & 1 & 1 & 1 & 1 & 4 \\
\hline Juvenile & Female & HiP 623 & 1 & & 1 & & 2 \\
\hline Juvenile & Female & Tembe 2 & 1 & 1 & 1 & 1 & 4 \\
\hline Juvenile & Female & Tembe 3 & 1 & 1 & 1 & 1 & 4 \\
\hline Juvenile & Male & HiP 407 & 1 & 1 & 1 & & 3 \\
\hline Juvenile & Male & HiP 408 & 1 & 1 & 1 & 1 & 4 \\
\hline Juvenile & Male & HiP 621 & 1 & & 1 & 1 & 3 \\
\hline Juvenile & Male & Tembe 1 & 1 & 1 & 1 & 1 & 4 \\
\hline Juvenile & Male & Tembe 6 & 1 & 1 & 1 & 1 & 4 \\
\hline Total & & & 30 & 26 & 40 & 36 & 132 \\
\hline
\end{tabular}

(GPA) that superimposes all the specimens to a common coordinate system by removing variation due to differences in position, scale and orientation (Rohlf \& Slice, 1990; Zelditch et al., 2012) (Fig. 1c). Due to superimposition, the morphometric analysis can be applied directly to the procrustes coordinates.

\section{Data analysis}

We used the subsample containing 10 paws and 
Table 2. Details of the sampled tracks. 'FL' = front left, 'FR' = front right, 'HL' = hind left and 'HR' = hind right.

\begin{tabular}{lllllrrrr}
\hline Trail number & Source of information & Age & Sex & FL & FR & HL & HR & Total \\
\hline 1 & Camera trap & Adult & Female & 5 & 1 & 6 & 4 & 16 \\
2 & Camera trap & Adult & Male & 2 & 1 & 3 & 3 & 9 \\
3 & Camera trap & Adult & Male & 1 & 1 & 4 & 3 & 9 \\
4 & Direct observation & Adult & Female & 2 & 7 & 6 & 7 & 22 \\
5 & Direct observation & Adult & Female & 2 & 4 & 3 & 3 & 12 \\
6 & Direct observation & Juvenile & NA & 2 & 2 & 2 & 2 & 8 \\
7 & Direct observation & Juvenile & NA & 2 & 3 & 4 & 3 & 12 \\
8 & Direct observation & Juvenile & NA & 2 & 2 & 2 & 1 & 7 \\
9 & Direct observation & NA & NA & 8 & 5 & 8 & 7 & 28 \\
10 & Direct observation & NA & NA & 8 & 8 & 7 & 8 & 31 \\
11 & Direct observation & NA & NA & 4 & 4 & 4 & 4 & 16 \\
12 & Direct observation & NA & NA & 3 & 3 & 3 & 3 & 12 \\
Total & & & & 41 & 41 & 52 & 48 & 182 \\
\hline
\end{tabular}

10 tracks to test the landmark digitization error by calculating the repeatability using repeatedmeasures nested ANOVA (Zelditch et al., 2012). Following a GPA and procrustes ANOVA to test the influence of the individual objects and four repetitions on shapes, we calculated the repeatability per manipulator using the equation (Zelditch et al., 2012):

$$
\begin{aligned}
\text { Repeatability }= & \text { Individual variance } /(\text { Mean } \\
& \text { Squares } \left._{\text {Repettions }}+\text { Individual variance }\right)
\end{aligned}
$$

with

$$
\begin{aligned}
\text { Individual variance }= & \left(\text { Mean Squares }_{\text {Individual }}-\right.\text { Mean } \\
& \text { Squares }_{\text {Repettions }} / \text { Number of repetitions }
\end{aligned}
$$

Secondly, we independently applied a GPA to (i) combination of paws and tracks, (ii) paws and (iii) tracks from the entire database. For each group, we ran a principal component analysis (PCA) on the procrustes coordinates. We used a procrustes ANOVA to test the influence of the factor object (i.e. paw or track) on the procrustes data combining paws and tracks, and the influence of the factor position (i.e. FR, HR, FL or $\mathrm{HL}$ ) on the procustes data of the paws and tracks independently. We applied a Linear Discriminant Analysis (LDA) with jack-knifed predictions and compared accuracy of the position prediction for paws and tracks by using an increasing number of principal components originating from the PCA on the procustes coordinates. Finally, we plotted the procrustes-aligned paws and tracks, together and independently, according to the first two principal components (i.e. PC1 versus PC2) and drew the thin-plate spline deformation grids representing the shape at the extremes of the range of variability along the two axes. Probability values were considered statistically significant at $P \leq 0.05$.

\section{RESULTS}

\section{Landmark error and position identification}

The mean landmark digitization repeatability was $88.80 \pm 5.09 \%$ for the paws and $73.65 \pm$ $5.49 \%$ for the tracks. The interaction between the factor object and shape was significant (procrustes ANOVA, $F_{1,312}=103.36, P=0.001$ ). The factor position has a significant influence on both the shape of paws (procrustes ANOVA, $F_{3,128}=25.83$, $P=0.001$ ) and tracks (procrustes ANOVA, $F_{3,178}=$ 44.16, $P=0.001$ ). The first 13 and 14 principal components explain more than $90 \%$ of the variability between the paws and tracks, respectively. The position prediction accuracy using the LDA with jack-knifed predictions reached a maximum of $95.45 \%$ and $91.21 \%$ for paws and tracks, respectively (Table 3 ).

\section{Shape variation between paws and tracks, between paws and between tracks}

$P C 1$, explaining $30.75 \%$ of the variation, displays a significant discriminating power between paws and tracks (Fig. 2a). Deformation grids show the main difference between paws and tracks to be the split of the toes (Fig. 2a). When paws and tracks are analysed separately, PC1 discriminates right from left, while PC2 discriminates front from hind (Fig. 2b,c). PC1 and PC2 explain 33.50\% and $15.27 \%$ of the variability between paws, and $35.32 \%$ and $16.32 \%$ of the variability between tracks. Considering that the dewclaw (located 
Table 3. Accuracy of position prediction (\%) for the paws and tracks using an increasing number of principal components in the linear discriminant analysis (LDA) with jack-knifed predictions. The maximum number of principal components used represents $>90 \%$ of the cumulative proportion of the variance, viz. 13 and 14 principal components for paws and tracks, respectively.

\begin{tabular}{ccc}
\hline \multirow{2}{*}{$\begin{array}{c}\text { Number of principal } \\
\text { components }\end{array}$} & \multicolumn{2}{c}{ Accuracy of prediction (\%) } \\
\cline { 2 - 3 } & Paws & Tracks \\
\hline 1 & 48.48 & 54.40 \\
2 & 84.09 & 88.46 \\
3 & 88.64 & 89.01 \\
4 & 88.64 & 88.46 \\
5 & 90.91 & 87.91 \\
6 & 90.91 & 87.91 \\
7 & 92.42 & 88.46 \\
8 & 91.67 & 89.01 \\
9 & 94.70 & 90.11 \\
10 & 94.70 & 91.21 \\
11 & 94.70 & 89.01 \\
12 & 95.45 & 88.46 \\
13 & 93.94 & 89.01 \\
14 & NA & 89.01 \\
\hline
\end{tabular}

higher up on the inner side of the leg) is not represented on paws and tracks, the lateral position is identified by the small digit (i.e. little finger or toe that is located on the outside of the leg) and the middle digit (i.e. between small digit and dewclaw, located two digits away from the small digit) (Fig. 2b,c). The small digit is shorter and positioned closer to the main pad. The middle digit is placed further away from the main pad; therefore, the upper tip marks the highest point of the paw or track. Particularly in tracks, the inner bottom lobe of the main pad (i.e. opposite side from the small digit) tends to be positioned higher than the outer bottom lobe. The front paws and tracks are larger and rounder than the hind paws and tracks (Fig. 2b,c).

\section{DISCUSSION}

Using 12 fixed landmarks, African lion paws and tracks present shapes that are complex enough to accurately and consistently identify their position

Fig. 1. Digital 3D model of (a) paw and (b) track with 12 fixed landmarks, and (c) procrustes coordinates of paws (132 specimens) and tracks (182 specimens). Note the two different landmark positioning sequences due to rotation symmetry between paw and track. In the procrustes coordinates, the specimens (grey points) surround the mean shape points. Links between the mean shape points were added for better visualization of the main pad and four toes.
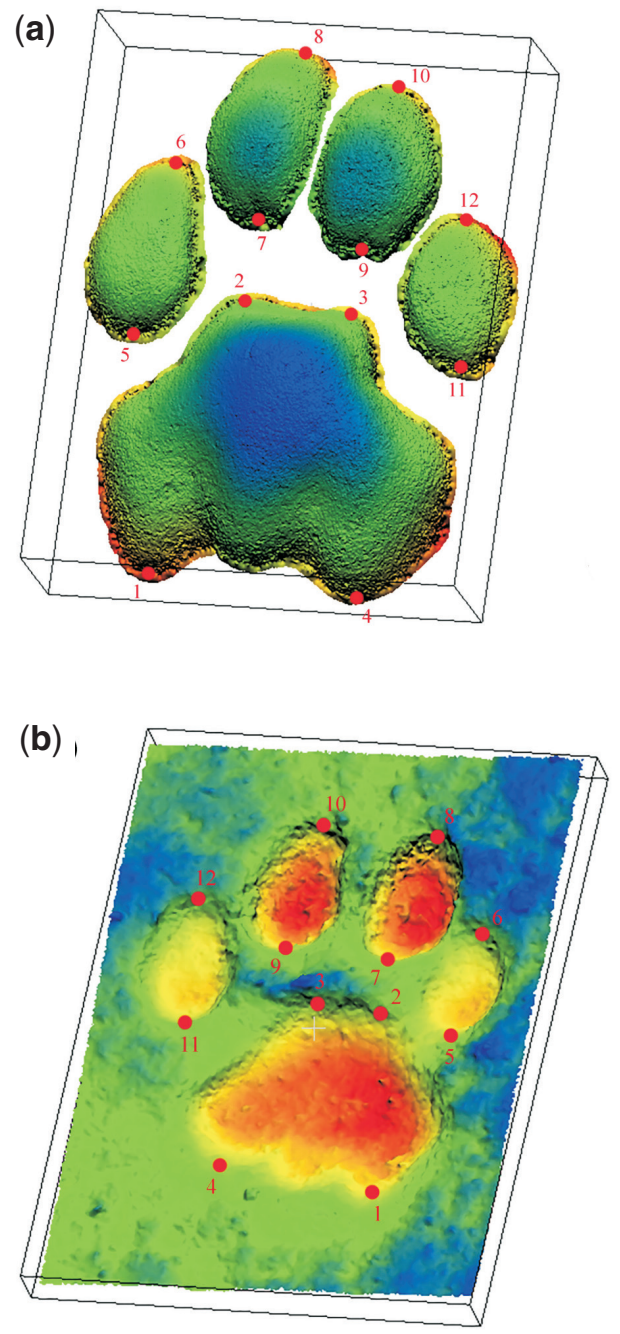

(c)

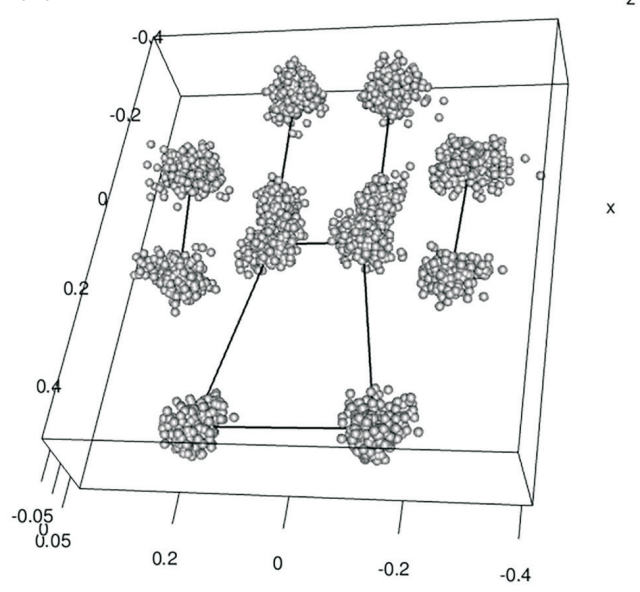



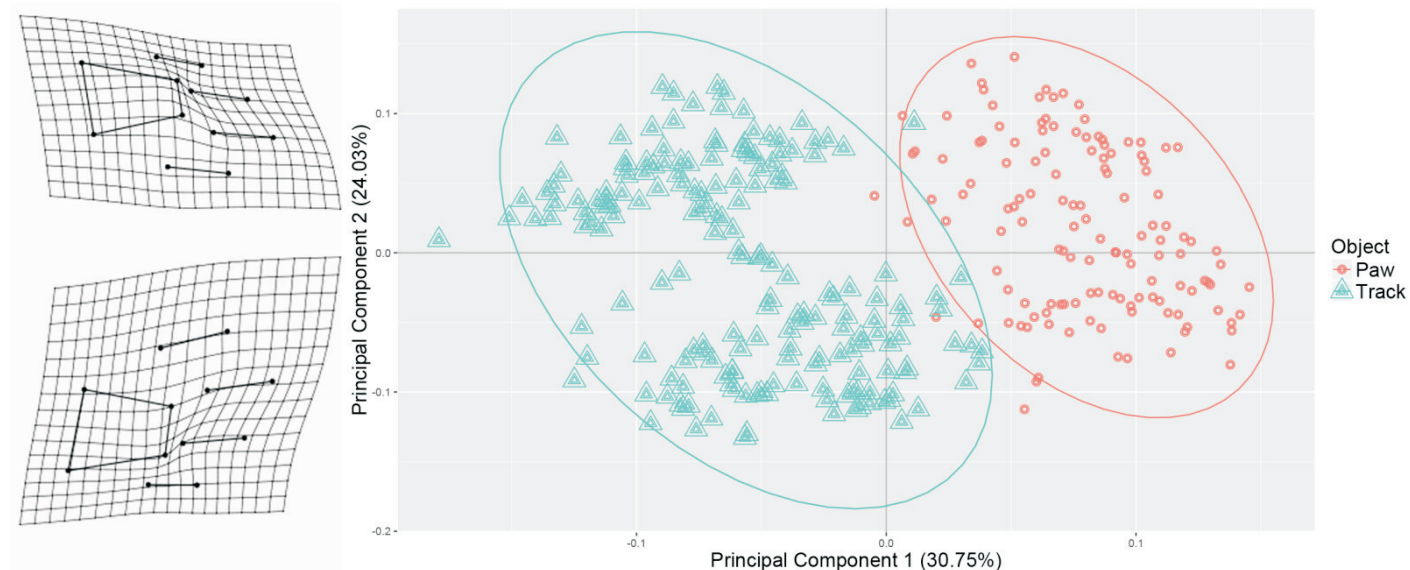

(a)
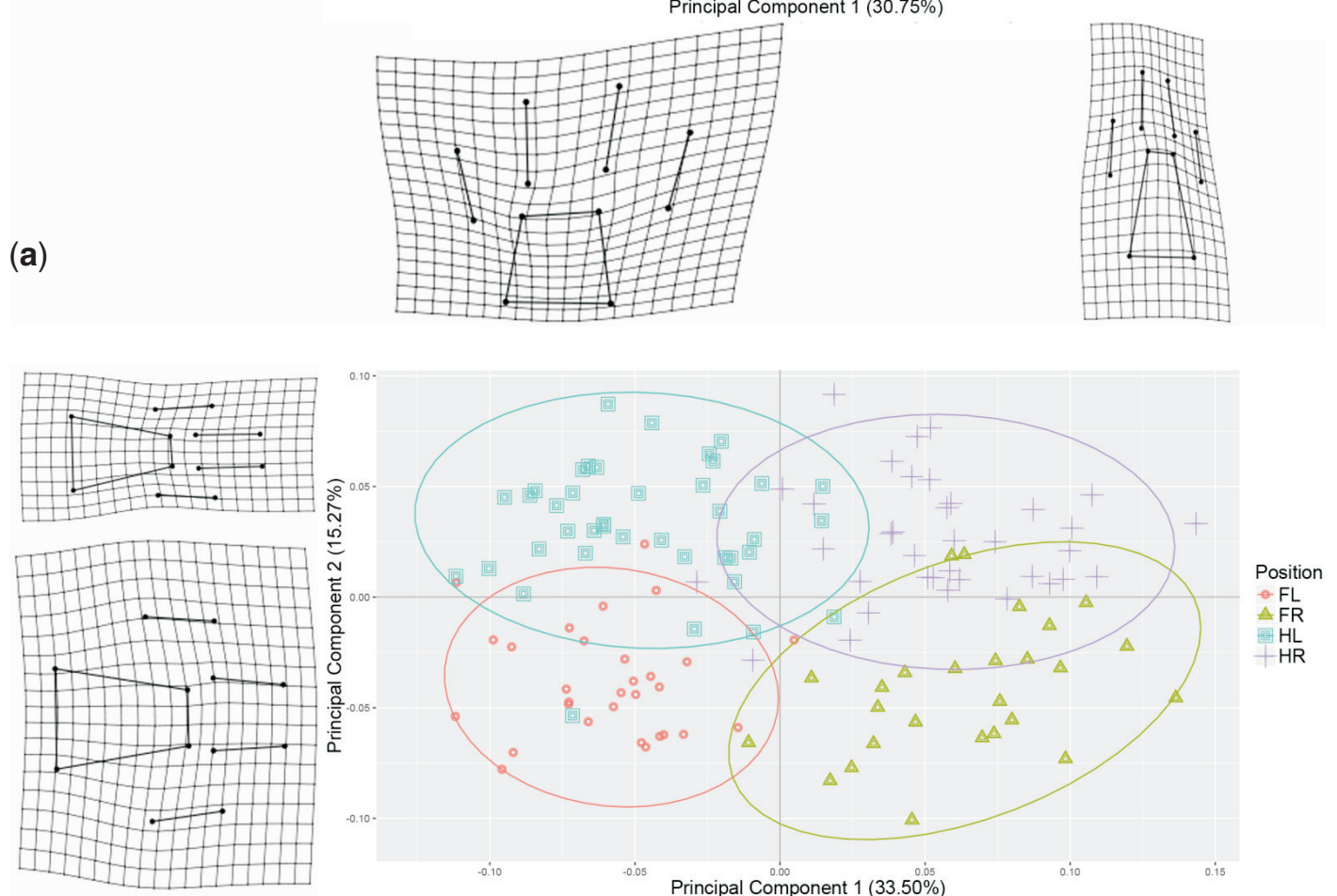

(b)
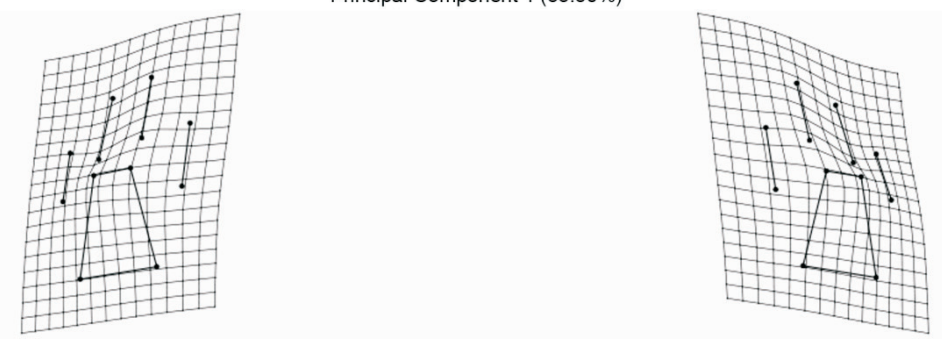

Fig. 2. Principal components of shape variation between (a) paws and tracks, (b) paws and (c) tracks. The thin-plate spline deformation grids (with 1.5 magnification) show the shape difference between extremes of each principal component axis and mean shape. Shape and colour code indicates the object (i.e. paw or track) (a) or the position (b and c). 'FL' = front left, 'FR' = front right, 'HL' = hind left and 'HR' = hind right. The ellipses represent the 95\% confidence intervals.

(Continued on p. 112) 


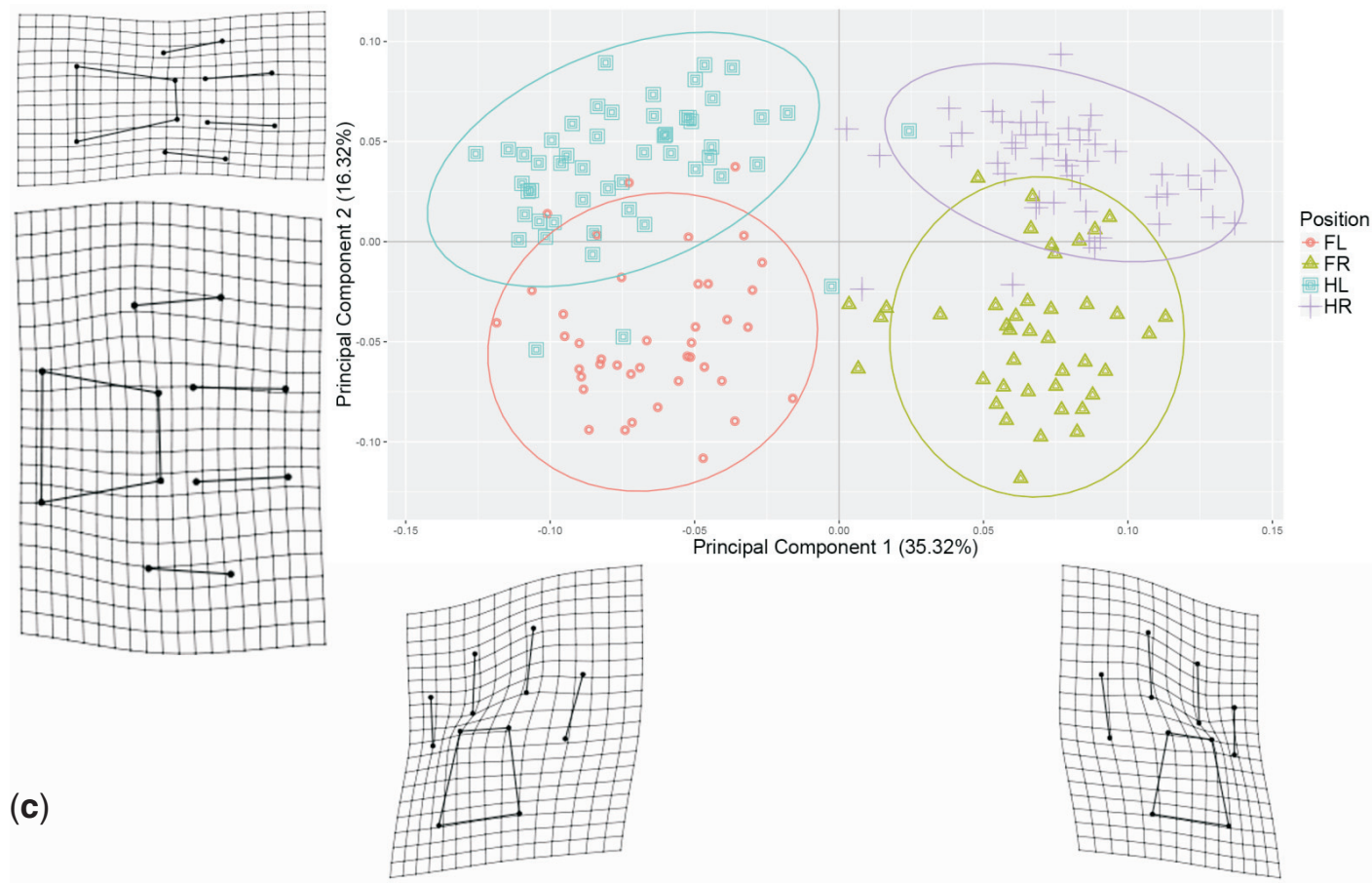

Fig. 2 (continued)

along both anteroposterior and mediolateral axes. A simple photogrammetric application enabled their shape recording in 3D, while geometric morphometrics permitted the study of shape variation and its covariation with other variables. The position identification from the paws yielded higher levels of prediction $(95.45 \%)$ than for the tracks $(91.21 \%)$ probably due to the distortion created by the complex interaction between the paw and substrate. This distortion seems to be more significant in the front tracks as a higher number of front tracks $(80 \%$ of the total missing tracks) were discarded from the analyses due to poor quality (i.e. the position of at least one of the 12 landmarks was missing or distorted). This can be explained by the fact that lions (as with most quadruped species) carry the majority of their weight on their front legs.

It is important to note that this research was based on tracks of relatively good quality (i.e. sampled in riverbeds and on sandy roads). We encourage future studies to analyse tracks sampled across different substrates, thus, resulting in differences in track quality. We also believe that the 3D information should be used to automate the segmentation and landmark positioning process. In order to extract a maximum amount of informa- tion from paws and tracks, to identify the age, sex and individual, we are currently investigating the use of more fixed landmarks along with curve- and surface-slider semi-landmarks. Furthermore, we wish to test the manipulator bias linked to the recording technique as well as the possibility of using a variety of digital cameras (ranging in price and quality) and different photogrammetric packages (including freeware).

The field equipment required to $3 \mathrm{D}$ record tracks for this study comprised a commercially available digital camera and scale bar. The simple and straightforward sampling protocol could enable the involvement of citizen-scientists and community members. Due to the affordability of equipment, easy field deployment and minimal need for logistical support, photogrammetry offers an ideal tool for longitudinal data sampling in remote and resourceless areas. This is on the premise that a third party handles the 3D processing step, feature extraction and statistical analysis (e.g. through the use of an online platform). We recommend the use of this objective position identification algorithm in future studies aiming to compare tracks from different lions. Identifying individuals from their tracks represents a practical low-cost non-invasive monitoring tool that would have 
major implications in the development of conservation, management and research strategies.

\section{ACKNOWLEDGEMENTS}

We thank EKZNW, particularly Dave Druce and Cathariné Hanekom, for allowing our research to be conducted at both study sites. Our appreciation goes to the Carnegie Institution for Science for allowing us to opportunistically utilize the tracking frequencies of collared individuals. We are grateful to Bronwen Klaas for proofreading earlier versions of this manuscript, and to Colette de Villiers, Fabien Démares, Henriek Bosua and Martin Postma for participating in the repeatability test. We thank the National Research Foundation (NRF) and the Rufford Foundation for funding parts of this research.

\section{REFERENCES}

Adams, D.C. \& Otárola-Castillo, E. (2013). geomorph: an $R$ package for the collection and analysis of geometric morphometric shape data. Methods in Ecology and Evolution, 4(4), 393-399.

Adams, D.C., Collyer, M.L. \& Sherratt, E. (2015). geomorph: software for geometric morphometric analyses. R package version 2.1.7. Retrieved from: http://cran.r-project.org/web/packages/geomorph /index.html on 22 September 2015.

Bennett, M. R., Falkingham, P., Morse, S. A., Bates, K. \& Crompton, R. H. (2013). Preserving the impossible: conservation of soft-sediment hominin footprint sites and strategies for three-dimensional digital data capture. PLOS ONE, 8(4), e60755.

Bookstein, F.L. (1991). Morphometric tools for landmark data: geometry and biology. Cambridge, U.K.: Cambridge University Press.

Cardillo, M., Purvis, A., Sechrest, W., Gittleman, J.L., Bielby, J. \& Mace, G.M. (2004). Human population density and extinction risk in the world's carnivores. PLOS Biology, 2(7), 0909-0914.

Ceballos, G., Ehrlich, P.R., Barnosky, A.D., García, A., Pringle, R.M. \& Palmer, T.M. (2015). Accelerated modern human-induced species losses: entering the sixth mass extinction. Science Advances, 1(5), e1400253.

Grigione, M.M., Burman, P., Bleich, V.C. \& Pierce, B.M. (1999). Identifying individual mountain lions Felis concolor by their tracks: refinement of an innovative technique. Biological Conservation, 88(1), 25-32.

Heinemeyer, K.S., Ulizio, T.J. \& Harrison, R.L. (2008). Natural sign: tracks and scats. In R.A. Long, P. MacKay, W.J.Zielinski \& J.C. Ray (Eds.), Noninvasive survey methods for carnivores (pp. 45-74). Washington, U.S.A.: Island Press.

Jewell, Z.C., Alibhai, S.K. \& Evans, J.W. (2014). Monitoring mountain lion using footprints: a robust new technique. Wild Felid Monitor, 7(1), 26-27.

Jewell, Z.C., Alibhai, S.K., Weise, F., Munro, S., Van Vuuren, M. \& Van Vuuren, R. (2016). Spotting cheetahs: identifying individuals by their footprints. Journal of Visualized Experiments, (111), e54034e54034.

Karanth, K.U., Nichols, J.D., Seidenstricker, J., Dinerstein, E., Smith, J.L.D., McDougal, C., Johnsingh, A.J.T., Chundawat, R.S. \& Thapar, V. (2003). Science deficiency in conservation practice: the monitoring of tiger populations in India. Animal Conservation, 6(2), 141-146.

Keating, A. P., Knox, J., Bibb, R. \& Zhurov, A. I. (2008). A comparison of plaster, digital and reconstructed study model accuracy. Journal of Orthodontics, 35(3), 191-201.

Liebenberg, L. (1990). A field guide to the animal tracks of southern Africa. Claremont, South Africa: David Philip.

Liebenberg, L., Louw, A. \& Elbroch, M. (2010). Practical tracking: a guide to following footprints and finding animals. Mechanicsburg, U.S.A.: Stackpole Books.

Marchal, A.F.J., Lejeune, P. \& De Bruyn, P.J.N. (2016). Virtual plaster cast: digital 3D modelling of lion paws and tracks using close-range photogrammetry. Journal of Zoology, 300(2), 111-119.

R Development Core Team (2014). R: a language and environment for statistical computing. Reference index version 3.2.2. Retrieved from: http://www R-project.org/ on 14 August 2015.

Riordan, P. (1998). Unsupervised recognition of individual tigers and snow leopards from their footprints. Animal Conservation, 1(04), 253-262.

Rohlf, F.J. \& Slice, D. (1990). Extensions of the Procrustes method for the optimal superimposition of landmarks. Systematic Biology, 39(1), 40-59.

Sharma, S., Jhala, Y. \& Sawarkar, V.B. (2005). Identification of individual tigers (Panthera tigris) from their pugmarks. Journal of Zoology, 267(1), 9-18.

Smallwood, K. S. \& Fitzhugh, E. L. (1993). A rigorous technique for identifying individual mountain lions Felis concolor by their tracks. Biological Conservation, 65(1), 51-59.

Yoccoz, N.G., Nichols, J.D. \& Boulinier, T. (2001). Monitoring of biological diversity in space and time. Trends in Ecology and Evolution, 16(8), 446-453.

Zelditch, M.L., Swiderski, D.L. \& Sheets, H.D. (2012). Geometric morphometrics for biologists: a primer (2nd edin). Amsterdam, Netherlands: Academic Press. 